\title{
Elastic nucleon form factors
}

\author{
M. De Sanctis \\ INFN, Sezione di Roma1, Piazzale Aldo Moro, Roma (Italy) \\ and \\ Universidad Nacional de Colombia, Bogotà (Colombia) \\ M.M. Giannini, E. Santopinto, A. Vassallo, \\ Dipartimento di Fisica dell'Università di Genova \\ and \\ Istituto Nazionale di Fisica Nucleare, Sezione di Genova, Italy,
}

\begin{abstract}
The relativized hypercentral Constituent Quark Model is used for the calculation of the elastic electromagnetic form factors of the nucleon. The results are compared with the recent measurements at Jlab.
\end{abstract}

A renewed interest in the electromagnetic form factors of the nucleon has been triggered by the recent results of the Jefferson Laboratory on the ratio between the electric and magnetic form factors of the proton [1. At variance with the expectations, the ratio deviates strongly from 1 and, for $Q^{2} \geq 1(\mathrm{GeV} / \mathrm{c})^{2}$, it decreases with an almost linear behaviour, pointing towards the possible existence of a zero at $Q^{2} \approx 8(\mathrm{GeV} / \mathrm{c})^{2}$. The main problem is the physical picture emerging from the data, that is the origin of the decrease of the ratio and of the eventual presence of a zero in the electric form factor. The first seems to be the manifestation of relativistic effects [2, 3, 4, while the possible presence or not of a zero will help to discriminate among the different models for the nucleon structure 15, 6, 2, 3, 7. For reviews on this subject the readers are referred to [8. Future experiments at higher $Q^{2}$ will clarify these points. From the theoretical point of view a zero in the proton charge form factor is a challenge for most models of the internal proton structure. In this contribution we report the results of recent calculations of the elastic nucleon form factors within a semirelativistic version of the hypercentral Constituent Quark Model (hCQM) [9].

The three quark hamiltonian for the hCQM is 9 .

$$
H=\frac{\vec{p}_{\rho}^{2}}{2 m}+\frac{\vec{p}_{\lambda}^{2}}{2 m}-\frac{\tau}{x}+\alpha x+H_{h y p},
$$

where $\vec{p}_{\rho}$ and $\vec{p}_{\lambda}$ are the conjugate momenta of the Jacobi coordinates $\vec{\rho}$ and $\vec{\lambda}$ and $x=\sqrt{\vec{\rho}^{2}+\vec{\lambda}^{2}}$ is the hyperradius. The spectrum is described with 
$\tau=4.59$ and $\alpha=1.61 \mathrm{fm}^{-2}$ and the standard strength of the hyperfine interaction needed for the $N-\Delta$ mass difference [10]. The $S U(6)_{s f}$ invariant potential is assumed to be of the type linear plus Coulomb-like, a form which is supported not only by the success of the the Cornell potential in the meson sector, but also by recent Lattice QCD calculations [11] for $S U(3)_{f}$ invariant static sources both for the meson and the baryon sectors. The model has been used for the prediction of various physical quantities of interest, namely the photocouplings [12, the electromagnetic transition amplitudes [13, the elastic nucleon form factors [14. The ratio between the electric and magnetic proton form factors [4] has been calculated boosting the three quark nucleon states to the Breit frame and expanding the matrix elements of the three quark current up to the first order in the quark momemtum. The non relativistic calculations predict the value $R=\mu_{p} \frac{G_{E}^{p}}{G_{M}^{p}}=1$ and introducing the hyperfine interaction makes no difference $(R=0.99)$. However, the first order relativistic corrections [4] give rise to a ratio which significantly deviates from 1 .

We have proposed a semirelativistic version of this model, introducing the relativistic kinetic energy:

$$
H=\sum_{i=1}^{3} \sqrt{\vec{k}_{i}^{2}+m_{i}^{2}}-\frac{\tau}{x}+\alpha x+H_{h y p}
$$

where $\vec{k}_{i}$ is the i-th quark 3 -momentum in the rest frame, i.e. $\sum_{i=1}^{3} \vec{k}_{i}=0$ and $m_{i}$ are the masses of the constituent quarks. This hamiltonian is solved by means of variational tecniques in the nucleon rest frame [15] and the eigenvalues fitted to the reproduction of the existing data for the 3 and 4 star resonances. The eigenfunctions of Eq. (2) are interpreted as the eigenfunctions of a mass operator in a Bakamjian-Thomas construction in a point form dynamics [16, 17. The nucleon states in the Breit frame are obtained from the rest ones by means of canonical boosts, which are purely kinematical and allow the composition of the angular momentum states as in the non relativistic case [17.

The nucleon electromagnetic form factors can be extracted from the matrix elements of the nucleon electromagnetic current according to the formalism described in [17, 3]. The electromagnetic current is written in impulse approximation, as already done by other authors [3] i.e. is chosen to be the sum of one-body quark currents [14, 4]:

$$
J_{\mu}^{(N)}=\sum_{i=1}^{3} e_{i} \gamma_{\mu}(i)
$$

where $e_{i}$ is the electric charge of the i-th constituent quark. The theoretical predictions are in quite a good agreement with the experimental data. Considering that constituent quarks have a finite size [18, we have introduced constituent quark form factors. The free parameters in the quark form factors have been fitted to the ratio R, the proton magnetic form factor $G_{M}^{p}$, the neutron electric $G_{E}^{n}$ and magnetic $G_{M}^{n}$ form factors [7], obtaining a very good reproduction of 
the data. As an example, the results for the ratio $\mathrm{R}$ are shown in Fig. 1. In Fig. 2 and 3 the results of the hCQM for the asymptotic behaviour are reported, without and with the constituent quark form factors, respectively. Perturbative QCD (pQCD) predicts 22 that at high $Q^{2}$ the helicity conserving Dirac form factor $F_{1 p} \propto \frac{1}{Q^{4}}$ and the helicity-flipping Pauli form factor $F_{2 p} \propto \frac{1}{Q^{6}}$, so that $Q^{2} \frac{F_{2 p}}{F_{1 p}}$ should reach a constant value at high enough $Q^{2}$. The asymptotic regime has not been reached yet (see the experimental data). Ralston et al. showed that if one takes into account the contributions from the non zero orbital angular momentum in the proton wave function, $\frac{F_{2 p}}{F_{1 p}}$ goes as $\frac{1}{Q}[23$ and this kind of scaling behaviour is reported in the right side of Fig.2 and 3. We observe that the experimental data for the ratios of Fig. 2 and Fig. 3 are quite well reproduced by the theoretical calculations. As a conclusion we can say that an extension of the measurement of the form factors to higher $Q^{2}$ values is important in order to test the pQCD scaling predictions for the Dirac and the Pauli form factors $F_{1 p}$ and $F_{2 p}$ and in particular to understand at which scale this behaviour starts. Moreover, a presence or not of a zero in the ratio of the electric and magnetic form factors of the proton, a clearly non perturbative effect, will help to discriminate among the different models of the nucleon.

\section{References}

[1] M.K. Jones et al., Phys. Rev. Lett. 84(2000) 1398; O. Gayou et al. , Phys. Rev. C 64(2001) 038202; O. Gayou et al., Phys. Rev. Lett. 88(2002) 092301.

[2] M. R. Frank, B. K. Jennings and G. A. Miller, Phys. Rev. C 54 (1996) 920; G.A. Miller, Phys. Rev. C 66 (2002) 032201(R); F. Cardarelli and S. Simula, Phys. Rev. C 62 (2000) 065201.

[3] R. F. Wagenbrunn, S. Boffi, W. Klink, W. Plessas and M. Radici, Phys. Lett. B 511 (2001) 33; S. Boffi, L. Y. Glozman, W. Klink, W. Plessas, M. Radici and R. F. Wagenbrunn, Eur. Phys. J. A 14 (2002) 17.

[4] M. De Sanctis, M. M. Giannini, L. Repetto and E. Santopinto, Phys. Rev. C 62 (2000) 025208.

[5] F. Iachello, A. D. Jackson and A. Lande, Phys. Lett. B 43 (1973) 191; R. Bijker and F. Iachello, Phys. Rev. C 69 (2004) 068201.

[6] E. L. Lomon, Phys. Rev. C 66 (2002) 045501; G. Holzwarth, Z. Phys. A 356 (1996) 339; G. Holzwarth hep-ph/0201138. D. Merten, U. Loring, K. Kretzschmar, B. Metsch and H. R. Petry, Eur. Phys. J. A 14 (2002) 477; D. H. Lu, A. W. Thomas and A. G. Williams, Phys. Rev. C 57 (1998) 2628 ;

J. D. Ashley, D. B. Leinweber, A. W. Thomas and R. D. Young, Eur. Phys. J. A 19 (2004) 9; B.Q.Ma, D.Qing and I. Schmidt, Phys. Rev. C 65, 035205 (2002). 
[7] M. De Sanctis, M. M. Giannini, E. Santopinto and A. Vassallo, nuclth/0506033.

[8] H. Y. Gao, Int. J. Mod. Phys. E 12 (2003) 1 [Erratum-ibid] E 12 (2003) 567]; K. De Jager and C. Hyde-Wright, Ann. Rev. Nucl. Part. Phys. 54 (2004) 1; B. Plaster et al. [Jefferson Laboratory E93-038 Collaboration], nucl-ex/0511025.

[9] M. Ferraris, M.M. Giannini, M. Pizzo, E. Santopinto and L. Tiator, Phys. Lett. B364 (1995) 231.

[10] N. Isgur and G. Karl, Phys. Rev. D18, 4187 (1978); Phys. Rev. D19 (1979) 2653.

[11] G. Bali et al., Phys. Rev. D 51 (1995) 5165; G. Bali, Phys. Rept. 343 (2001) 1; C. Alexandrou, P. de Forcrand and O. Jahn, Nucl. Phys. Proc. Suppl. 119 (2003) 667; H. Suganuma, T. T. Takahashi, F. Okiharu and H. Ichie, Nucl. Phys. Proc. Suppl. 141 (2005) 92.

[12] M. Aiello, M. Ferraris, M.M. Giannini, M. Pizzo and E. Santopinto, Phys. Lett. B387 (1996) 215.

[13] M. Aiello, M. M. Giannini, E. Santopinto, J. Phys. G: Nucl. Part. Phys. 24 (1998) 753.

[14] M. De Sanctis, E. Santopinto, M.M. Giannini, Eur. Phys. J. A1 (1998) 187.

[15] M.M. Giannini, E. Santopinto, M. Traini, A. Vassallo, in preparation.

[16] B. D. Keister and W. N. Polyzou, Adv. Nucl. Phys. 20 (1991) 225.

[17] W. H. Klink, Phys. Rev. 58 (1998) 3587.

[18] R. Petronzio, S. Simula and G. Ricco, Phys. Rev. D 67 (2003) 094004.

[19] B. D. Milbrath et al. [Bates FPP collaboration], Phys. Rev. Lett. 80 (1998) 452 [Erratum-ibid. 82 (1999) 2221]

[20] T. Pospischil et al. [A1 Collaboration], Eur. Phys. J. A 12 (2001) 125.

[21] V. Punjabi et al., Phys. Rev. C 71 (2005) 055202 [Erratum-ibid. C 71 (2005) 069902].

[22] S. J. Brodsky and G. R. Farrar, Phys. Rev. Lett. 31 (1973) 1153; S. J. Brodsky and G. R. Farrar, Phys. Rev. D 11 (1975) 1309.

[23] J. P. Ralston and P. Jain, Phys. Rev. D 69053008 (2004). 


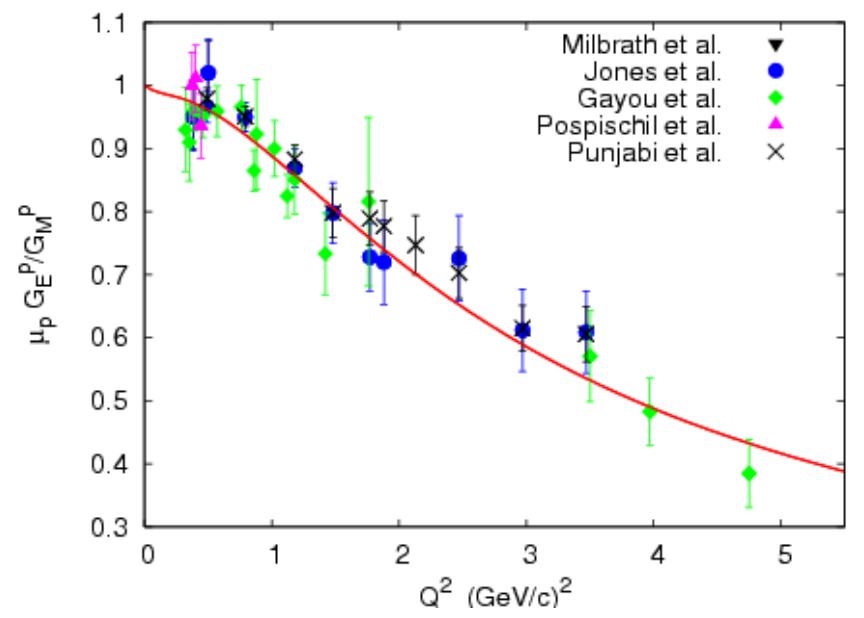

Figure 1: (Color online) The ratio $\mu_{p} \hat{G}_{E} / G_{M}^{p}$ from polarization transfer compared with the semirelativistic hCQM calculation with constituent quark form factors (solid line). The experimental data are taken from [19, 1, 20, 21]. 

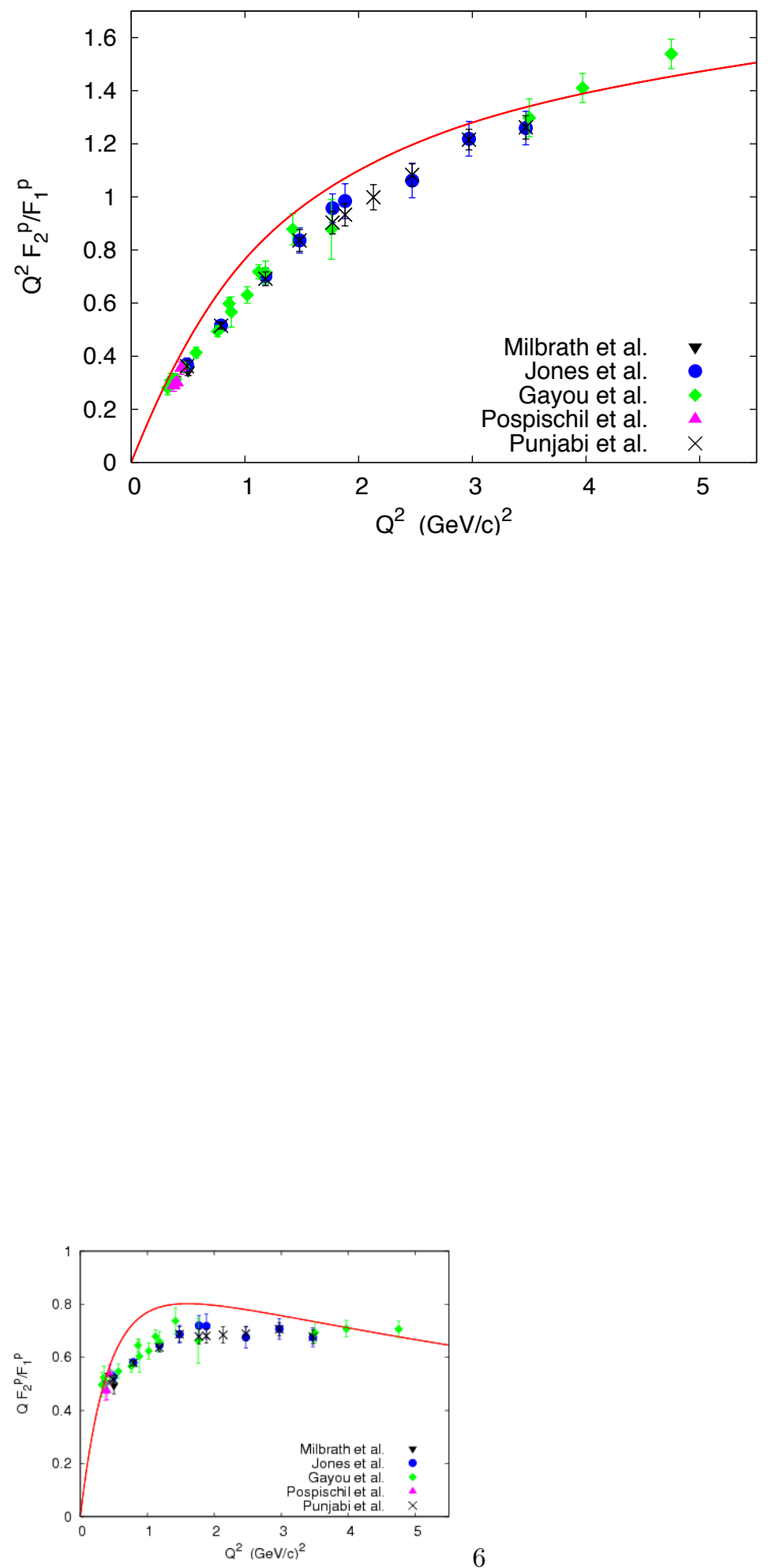

Figure 2: (Color on line) The ratio $Q^{2} \frac{F_{2 p}}{F_{1 p}}$ and $Q \frac{F_{2 p}}{F_{1 p}}$, calculated with the relativized hCQM (solid line). The experimental data are taken from $[19,20,21]$. 

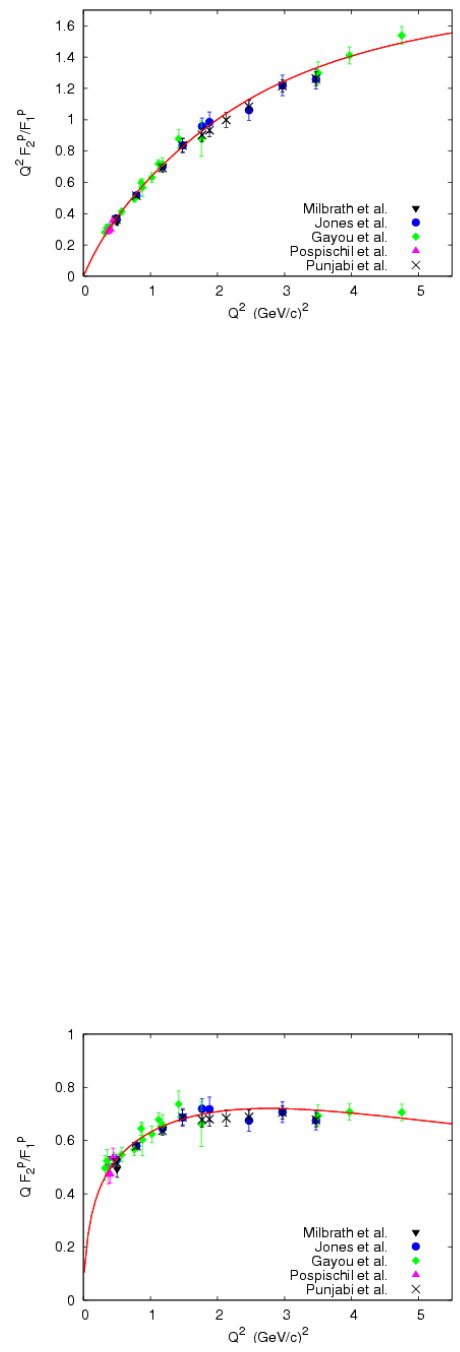

Figure 3: (Color on line)The ratio $Q^{2} \frac{F_{2 p}}{F_{1 p}}$ and $Q \frac{F_{2 p}}{F_{1 p}}$, calculated with the relativized hCQM with the inclusion of constituent quark form factors (solid line). The experimental data are taken from [19,20,21]. 